\title{
COLOUR IMAGE ENHANCEMENT BASED ON HISTOGRAM EQUALIZATION
}

\author{
Kanika Kapoor and Shaveta Arora \\ Department of ECE, ITM University, Gurgaon, Haryana, India
}

\begin{abstract}
Histogram equalization is a nonlinear technique for adjusting the contrast of an image using its histogram. It increases the brightness of a gray scale image which is different from the mean brightness of the original image. There are various types of Histogram equalization techniques like Histogram Equalization, Contrast Limited Adaptive Histogram Equalization, Brightness Preserving Bi Histogram Equalization, Dualistic Sub Image Histogram Equalization, Minimum Mean Brightness Error Bi Histogram Equalization, Recursive Mean Separate Histogram Equalization and Recursive Sub Image Histogram Equalization. In this paper, the histogram equalization approach of gray-level images is extended for colour images. The acquired image is converted into HSV (Hue, Saturation, Value). The image is then decomposed into two parts by using exposure threshold and then equalized them independently Over enhancement is also controlled in this method by using clipping threshold. For measuring the performance of the enhanced image, entropy and contrast are calculated.
\end{abstract}

\section{KEYWORDS}

Colour Enhancement, Histogram Equalization, Entropy, Contrast.

\section{INTRODUCTION}

Image Enhancement is the process of improving the visual quality of an image so that the results are more suitable than the original. The process of image Enhancement is divided into two domains i.e. frequency domain and spatial domain. In frequency domain, techniques operate on frequency transform of the image whereas in spatial domain, techniques operate directly on the pixels of the image [1]. Image Enhancement is used to improve the contrast of the image having low luminance. Image enhancement removes the distortion from the image and improves the quality of the image. Histogram Equalization is the enhancement technique. Histogram Equalization is used to enhance the contrast of the colour image. Histogram Equalization is the simple method for enhancing the quality of image. Histogram Equalization (HE) is the process of remaps the pixels of the image. Histogram Equalization distributes the pixels of the image on the dynamic range for enhancing the contrast of the image. There is various application of Histogram Equalization such as medical image processing, sonar image processing, object tracking, speech recognition, etc. For improving the contrast of the colour image Histogram Equalization is one of the techniques which are used. Brightness Preserving Bi-Histogram Equalization (BBHE) was proposed by kim in 1997 [2]. It divides the histogram of the image into two parts i.e. underexposed and overexposed based on the mean value. Then it equalizes the sub image 
histogram of original image. Dualistic Sub Image Histogram Equalization (DSIHE) was proposed by Wan et al. in 1999. It is also used for preserving the brightness of image based on median value not mean value [3]. In this method original histogram is divided into two parts based on median value and then it equalizes the sub image histograms independently. Minimum Mean Brightness Error Bi-Histogram Equalization (MMBEBHE) was proposed by Chen and Ramli in $2003 \mathrm{a}, \mathrm{b}$. It is the extension of brightness preserving bi-histogram equalization. In this we can measure the brightness of image by using Absolute Mean Brightness Error (AMBE) $[4,5]$. If the Absolute Mean Brightness Error is minimum then it can improve the contrast of the image and preserve the brightness of the image. Recursive Mean Separate Histogram Equalization was proposed by Chen and Ramli in 2003a,b. It divides the histogram recursively up to a recursion level $r$, generating $2^{r}$ sub-histograms based on the mean value and equalized them independently $[4,5]$. Recursive Sub Image Histogram Equalization recursively was proposed by Sim et al. in 2007 [6]. In this histogram is recursively divided based on their median value and equalize them independently.

In this paper we have extended the Exposure Based Sub Image Histogram Equalization for improving the contrast of the colour images. Some other histogram based techniques are compared with the proposed technique based on entropy and contrast of the image.

\section{HISTOGRAM EQUALIZATION}

Histogram Equalization is used to enhance the contrast of the colour image. It is one of the simplest and less complex methods for enhancing the quality of the colour image. It distributes the pixels of the image on the dynamic range for enhancing the contrast of the image [7]. It is the process that remaps the pixels of the image. For improving the contrast of the image following are the histogram based techniques [8] i.e.
A. Classical Histogram Equalization (CHE)
B. Contrast-limited adaptive histogram equalization (CLAHE)
C. Brightness Preserving Bi-Histogram Equalization (BBHE)
D. Dualistic Sub Image Histogram Equalization (DSIHE)
E. Minimum Mean Brightness Error Bi-Histogram Equalization (MMBEBHE)
F. Recursive Mean Separate Histogram Equalization (RMSHE)
G. Recursive Sub Image Histogram Equalization (RSIHE)
H. Exposure Based Sub Image Histogram Equalization (ESIHE).

\section{Classical Histogram Equalization}

Classical Histogram produces an equalized image with its fatten histogram. Histogram Equalization is used to enhance the contrast of the image. Histogram Equalization is the simple method for enhancing the contrast of image. Histogram Equalization distributes the pixels of the 
Electrical \& Computer Engineering: An International Journal (ECIJ) Volume 4, Number 3, September 2015

image on the dynamic range for enhancing the contrast of the image [7]. Basically this method uniformly distributes the gray level of the image; the range of gray level is 0 to L-1. So it improves the contrast of the image. But this method also has some disadvantage. While distributing the pixels on the dynamic range, signal gets distorted.

\section{CONTRASt Limited AdAPTIVE Histogram EqALiZation}

Adaptive Histogram Equalization is also use to enhance the image's contrast. In Adaptive Histogram equalization for improving the contrast of the image we divide the image into different small parts and take the histogram of each part and uses to redistributes the lightness values [8]. So the output of the adaptive histogram is same as the specified histogram. By using bilinear interpolation the other neighbouring small parts of the image are combined [9]. This method is different from Classical Histogram Equalization. The drawback of this method is that it cannot regain the brightness as the input image. So to overcome on this drawback we use advance version of adaptive histogram equalization i.e. Contrast Limited Adaptive Histogram Equalization which is also fail to regain the brightness of image.

\section{Exposure BaSed Sub Image Histogram Equalization}

Exposure Based Sub Image Histogram Equalization is also used for contrast enhancement [10]. It divides the image into two sub image i.e. under exposed and over exposed image based on the exposure threshold value

$$
\text { exposure }=\frac{1}{\mathrm{~L}} \frac{\sum_{k=1}^{L} \mathrm{~h}(\mathrm{k}) \mathrm{k}}{\sum_{k=1}^{L} \mathrm{~h}(\mathrm{k})}
$$

Where,

$$
\begin{aligned}
& \mathrm{L}=\text { total no of gray level } \\
& \mathrm{h}(\mathrm{k})=\text { histogram of the image }
\end{aligned}
$$

This exposure threshold is used for clipping histogram. The basic purpose for using clipping is to prevent the over enhancement.

$$
\begin{aligned}
& \mathrm{T}_{\mathrm{c}}=\frac{1}{L} \sum_{k=1}^{L} \mathrm{~h}(\mathrm{k}) \\
& \mathrm{h}_{\mathrm{c}}(\mathrm{k})=\mathrm{Tc} \text { for } \mathrm{h}(\mathrm{k}) \geq \mathrm{T}_{\mathrm{c}}
\end{aligned}
$$

Where,

$$
\begin{aligned}
& \mathrm{T}_{\mathrm{c}}=\text { clipping threshold } \\
& \mathrm{h}_{\mathrm{c}}(\mathrm{k})=\text { clipped histogram } \\
& \mathrm{h}(\mathrm{k})=\text { original histogram of the image }
\end{aligned}
$$

By using exposure threshold value we find the parameter $\mathrm{X}_{\mathrm{m}}$

$$
\mathrm{X}_{\mathrm{m}}=\mathrm{L}(1-\text { exposure })
$$


On the basis of this parameter we divide the histogram into sub histograms i.e. underexposed and overexposed. After diving we find their probability density function and cumulative density function respectively and equalize them independently.

$$
\begin{aligned}
& \mathrm{P}_{\mathrm{u}}(\mathrm{k})=\frac{\mathrm{h}_{\mathrm{c}}(\mathrm{k})}{\mathrm{N}_{\mathrm{u}}}, \text { for } 0 \leq \mathrm{k} \leq \mathrm{X}_{\mathrm{m}} \\
& \mathrm{P}_{\mathrm{o}}(\mathrm{k})=\frac{\mathrm{h}_{\mathrm{c}}(\mathrm{k})}{\mathrm{N}_{\mathrm{o}}}, \text { for } \mathrm{X}_{\mathrm{m}}+1 \leq \mathrm{k} \leq \mathrm{L}-1 \\
& \mathrm{C}_{\mathrm{u}}(\mathrm{k})=\sum_{\mathrm{k}=0}^{\mathrm{Xa}} \mathrm{P}_{\mathrm{u}}(\mathrm{k}) \\
& \mathrm{C}_{\mathrm{o}}(\mathrm{k})=\sum_{\mathrm{k}=\mathrm{Xa}+1}^{\mathrm{L}-1} \mathrm{P}_{\mathrm{o}}(\mathrm{k})
\end{aligned}
$$

Where,

$$
\begin{aligned}
& \mathrm{N}_{\mathrm{u}}=\text { total no of pixels in underexposed image } \\
& \mathrm{N}_{\mathrm{o}}=\text { total no of pixels in overexposed image } \\
& \mathrm{P}_{\mathrm{u}}(\mathrm{k})=\text { pdf of underexposed image } \\
& \mathrm{P}_{\mathrm{o}}(\mathrm{k})=\text { pdf of overexposed image } \\
& \mathrm{C}_{\mathrm{u}}=\text { cdf of underexposed image } \\
& \mathrm{C}_{\mathrm{o}}=\text { cdf of overexposed image }
\end{aligned}
$$

For equalization we use transfer function. After equalization combines the sub images and gives the output i.e. enhanced image.

$$
\begin{gathered}
\mathrm{F}_{\mathrm{u}}=\mathrm{X}_{\mathrm{m}} * \mathrm{C}_{\mathrm{u}} \\
\mathrm{F}_{\mathrm{o}}=\left(\mathrm{X}_{\mathrm{m}}+1\right)+\left(\mathrm{L}-\mathrm{X}_{\mathrm{m}}+1\right) \mathrm{C}_{\mathrm{o}}
\end{gathered}
$$

Where,

$$
\begin{aligned}
& \mathrm{F}_{\mathrm{u}}=\text { transfer function for equalizing underexposed image } \\
& \mathrm{F}_{\mathrm{o}}=\text { transfer function for equalizing overexposed image }
\end{aligned}
$$

This method cannot be directly applied to the colour image. First we have to convert the RGB component of colour image into HSV component and then extract the V component from the HSV component of the image. Now the given algorithm for colour enhancement is applied and finds the enhanced. Getting the enhanced colour image we combine the new V component with the $\mathrm{H}$ and $\mathrm{S}$ components and then convert the HSV component into RGB component.

This method is best method for preserving the brightness. It gives highest entropy. Exposure Based Sub Image is suitable for underexposed image. It controls the over enhancement by histogram clipping. It improves the quality and colour contrast of the image better than other methods. 


\section{Flow Chart}

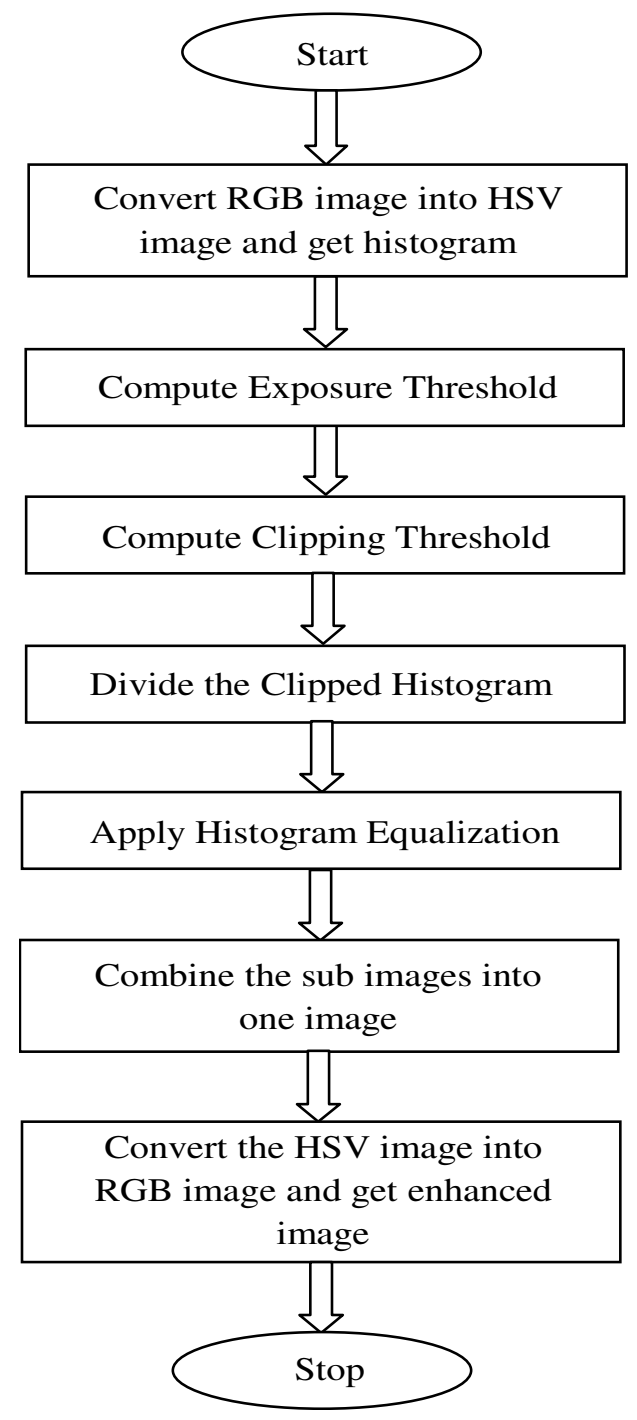

Fig1. Flow chart of technique

\section{Performance Measure}

For measuring the performance of the image we calculate two parameters i.e. entropy and contrast.

\subsection{Entropy}

Here, we use entropy as the Shannon entropy which contains the maximum information [10]. It is use to measure the quality of image. Images which have maximum entropy have the better quality of the image. 
Electrical \& Computer Engineering: An International Journal (ECIJ) Volume 4, Number 3, September 2015

Where,

$$
\operatorname{Ent}(\mathrm{p})=-\sum_{\mathrm{l}=0}^{\mathrm{L}-1} \mathrm{p}(l) \log l
$$

Ent $=$ entropy of the image.

$\mathrm{P}(l)=$ probability density function at intensity level 1 .

$\mathrm{L}=$ total no of gray level.

\subsection{Contrast}

Second parameter is contrast which is also used for measuring the quality of the image. Image which have highest value of contrast have good contrast visually.

where,

$$
\text { contrast }=\sum_{i, j}|i-j|^{2} p(i, j)
$$

$$
p(i, j)=\text { no of gray level occurrence }
$$

\section{Results AND Discussions}

The proposed method is simulated on Matlab 2010. The simulation results of HE and CLAHE are compared with the proposed method for eight colour images as shown in figure 2-9. For measuring the performance of the image we have calculated two parameters entropy and contrast. Table 1 shows the entropy values of all the images for different algorithms which are demonstrated in Fig. 10. Table 2 shows the contrast of all the images using different algorithm which is demonstrated in Fig.11. The visual results of the proposed method show more pleasing effects than the other methods. Proposed method is suitable for underexposed low contrast images. It gives the enhanced image with highest entropy and good contrast by reducing over enhancement. HE sometimes over enhances the image and CLAHE shows over amplification and some distorted pixel.

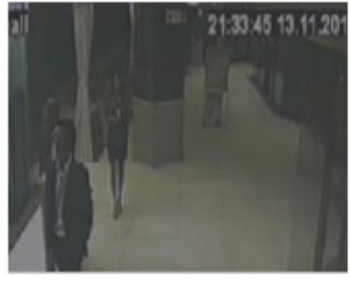

(a)

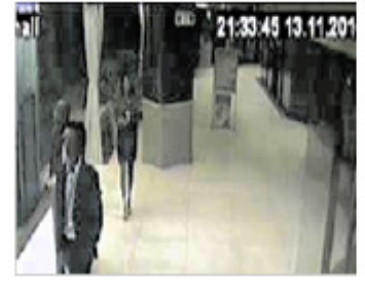

(b)

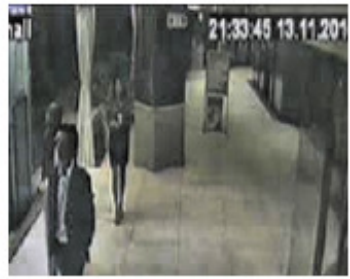

(c)

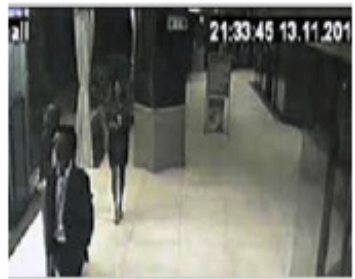

(d)

Fig 2. Enhancement results of CCTV image: (a) Original, (b) HE, (c) CLAHE, and (h) ESIHE.

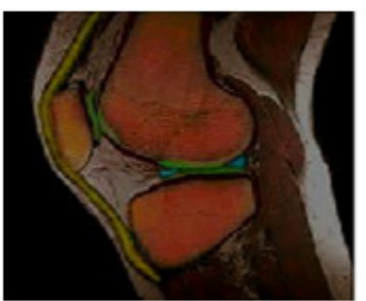

(a)

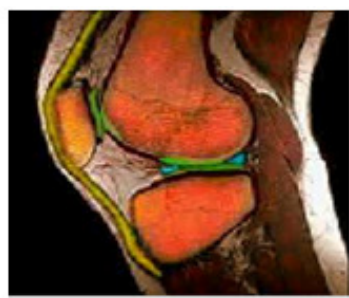

(b)

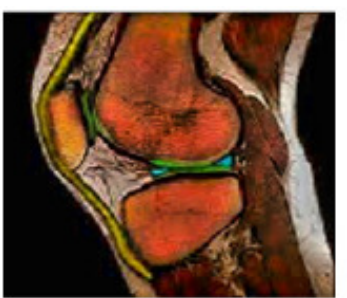

(c)

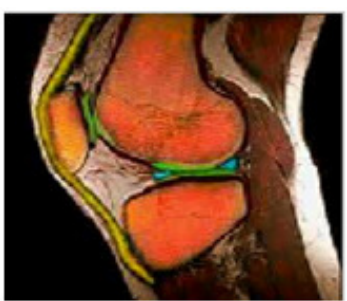

(d)

Fig 3. Enhancement results of Knee image: (a) Original, (b) HE, (c) CLAHE, and (h) ESIHE 
Electrical \& Computer Engineering: An International Journal (ECIJ) Volume 4, Number 3, September 2015

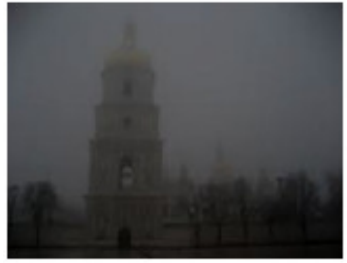

(a)

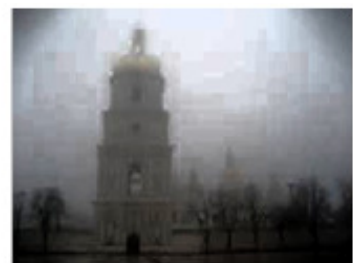

(b)

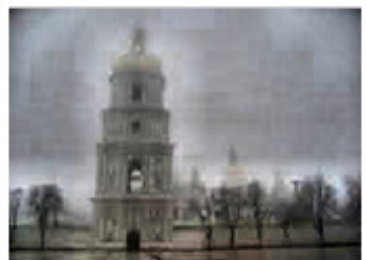

(c)

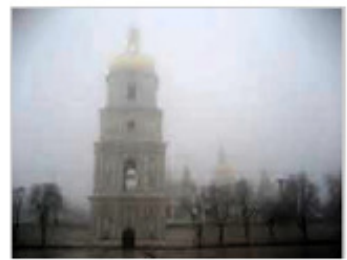

(d)

Fig 4. Enhancement results of Building image: (a) Original, (b) HE, (c) CLAHE, and (h) ESIHE.

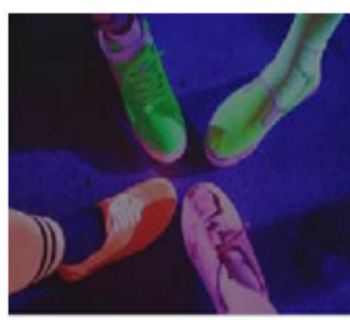

(a)

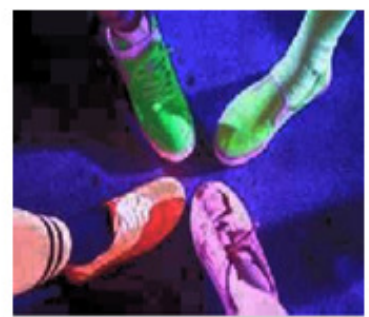

(b)

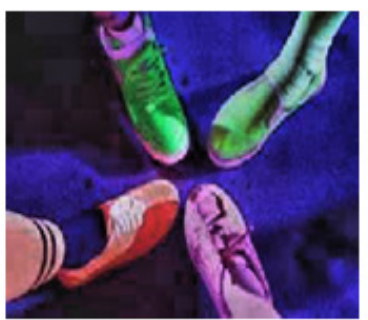

(c)

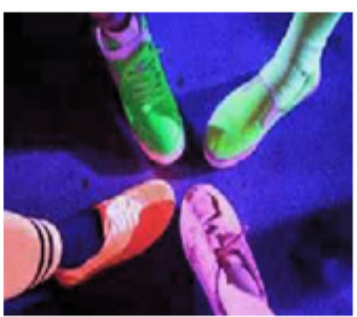

(d)

Fig 5. Enhancement results of Shoe image: (a) Original, (b) HE, (c) CLAHE, and (h) ESIHE

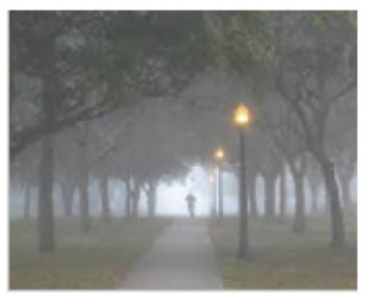

(a)

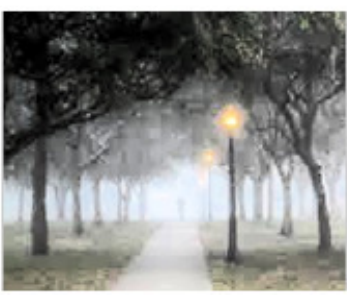

(b)

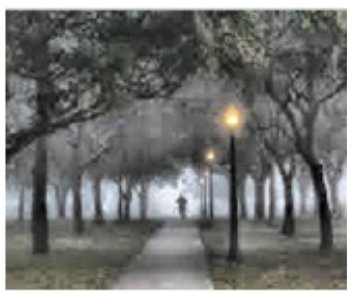

(c)

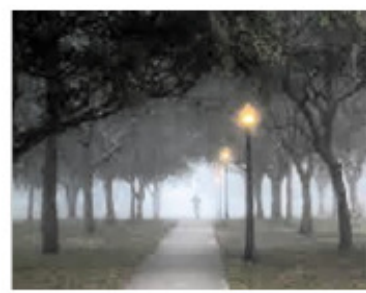

(d)

Fig 6. Enhancement results of Forest image: (a) Original, (b) HE, (c) CLAHE, and (h) ESIHE

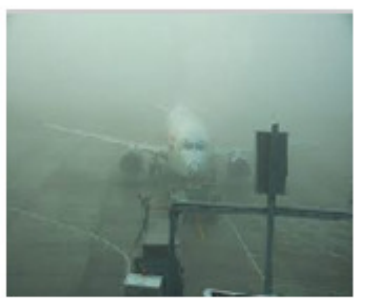

(a)

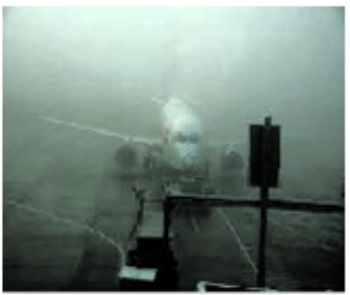

(b)

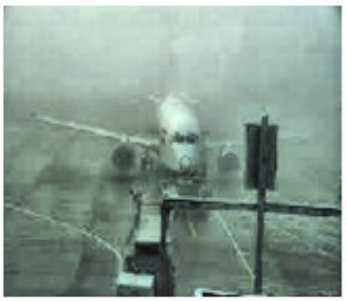

(c)

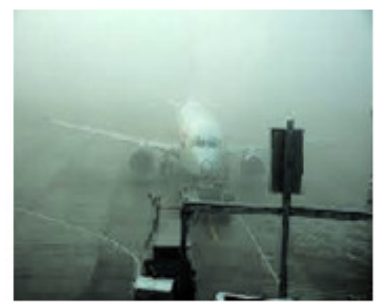

(d)

Fig 7. Enhancement results of Plane image: (a) Original, (b) HE, (c) CLAHE, and (h) ESIHE

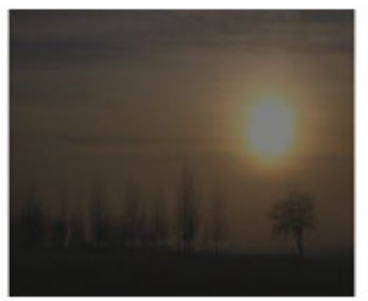

(a)

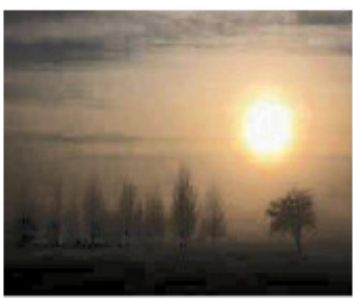

(b)

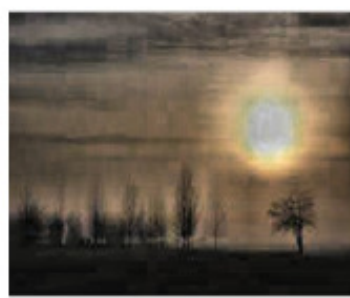

(c)

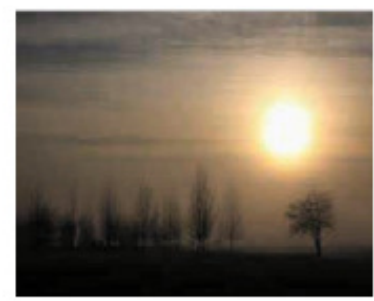

(d)

Fig 8. Enhancement results of Sun image: (a) Original, (b) HE, (c) CLAHE, and (h) ESIHE 
Electrical \& Computer Engineering: An International Journal (ECIJ) Volume 4, Number 3, September 2015

Figure 2(a)-8(a) are the original dark images which are required to enhance. Fig 2(b)-8(b) shows the simple histogram equalization. In histogram equalization over enhancement occurred. Fig2(c)-8(c) shows the contrast limited adaptive histogram equalization but this method cannot regain the brightness of the original image. Fig 2(d)-8(d) shows the result of the proposed method which gives the enhanced image. The entropy of the enhanced image from the proposed method is the highest than the other methods as calculated in Table 1. Contrast is also highest of the enhanced image as given in Table 2. It can remove the smoke from the image. The image of building, plane, and tree are the smoky images and our proposed method can remove the smoke and enhance the image better than the others. The average entropy of proposed method is higher than the average entropy of other methods.

Comparison of entropies of all the images is plotted for different methods in Fig.9. In this graph we can see that our proposed method has the maximum entropy than the other method. It has the richness in details than the original image. Fig 10 shows the contrast of all the images. In this also contrast from proposed method is the highest among all.

Table 1: Comparison of Entropy of all images.

\begin{tabular}{|c|c|c|c|c|}
\hline IMAGES & ORIGINAL & HE & CLAHE & ESIHE \\
\hline Building & 6.4161 & 7.3464 & 7.2442 & 7.4747 \\
\hline Plane & 7.2653 & 7.6586 & 7.5494 & 7.7936 \\
\hline CCTV & 6.1620 & 7.1711 & 7.3033 & 7.3962 \\
\hline Forest & 6.6172 & 7.4067 & 7.3023 & 7.4716 \\
\hline Sun & 6.3935 & 7.3513 & 7.1471 & 7.3617 \\
\hline Tree & 6.7911 & 7.5117 & 7.3754 & 7.5749 \\
\hline Shoe & 6.4953 & 7.1882 & 7.1616 & 7.3972 \\
\hline Knee & 5.8835 & 6.2203 & 6.7304 & 6.5018 \\
\hline
\end{tabular}

Table 2: Comparison of contrast of all images.

\begin{tabular}{|c|c|c|c|c|}
\hline IMAGES & ORIGINAL & HE & CLAHE & ESIHE \\
\hline Building & 0.0309 & 0.0636 & 0.1345 & 0.0673 \\
\hline Plane & 0.0438 & 0.0958 & 0.1838 & 0.1041 \\
\hline CCTV & 0.0885 & 0.7048 & 0.4423 & 0.4831 \\
\hline Forest & 0.0879 & 0.2590 & 0.3430 & 0.1932 \\
\hline Sun & 0.0256 & 0.0424 & 0.0955 & 0.0399 \\
\hline Tree & 0.0984 & 0.3641 & 0.4702 & 0.2346 \\
\hline Shoe & 0.0972 & 0.2830 & 0.2902 & 0.2426 \\
\hline Knee & 0.1962 & 0.4681 & 0.5654 & 0.4908 \\
\hline
\end{tabular}


Electrical \& Computer Engineering: An International Journal (ECIJ) Volume 4, Number 3, September 2015

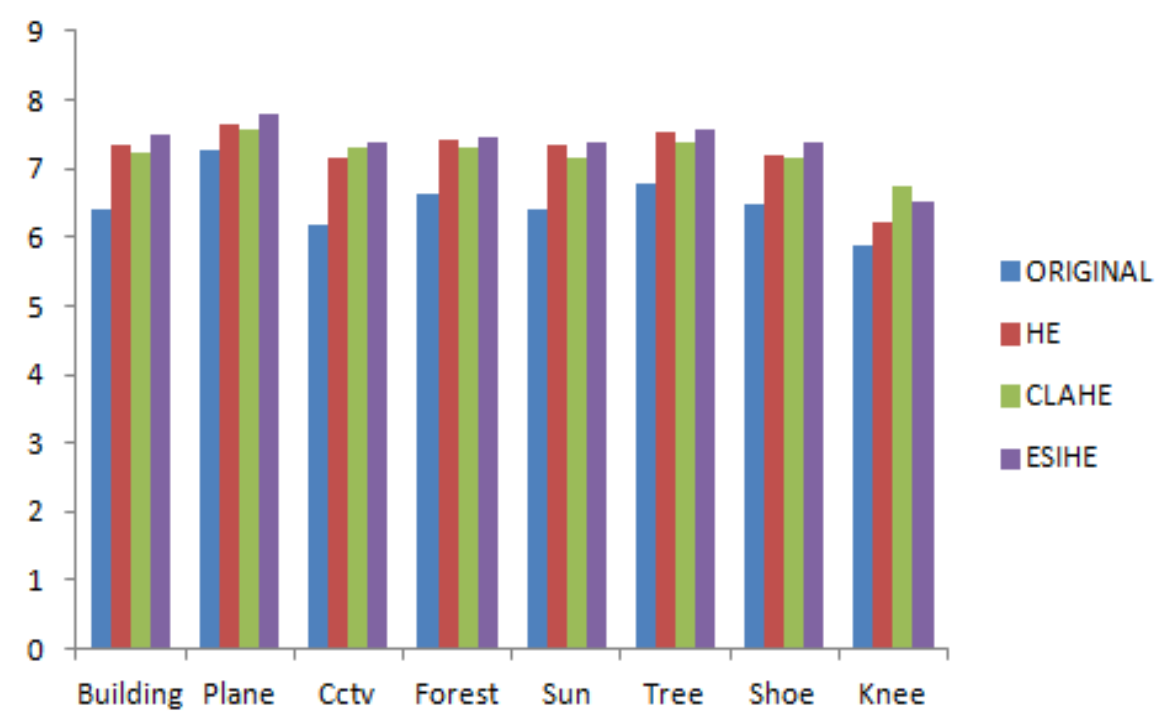

Fig 9. Entropy of all images

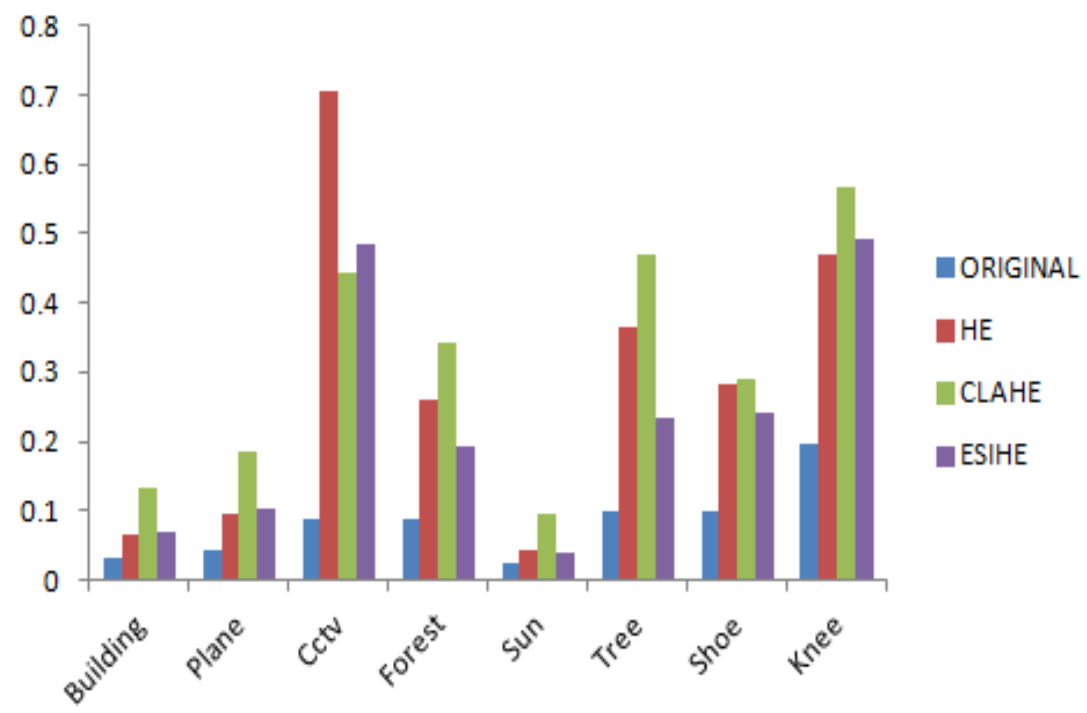

Fig 10 Contrast of all images

\section{Conclusion}

In this paper, histogram based technique is proposed for the enhancement of the underexposed color images. Entropy and contrast values are calculated for different low contrast images. For the enhancement of under exposed image this proposed method is effective and efficient. The results show better performance of this technique in comparison to others. 
Electrical \& Computer Engineering: An International Journal (ECIJ) Volume 4, Number 3, September 2015

\section{REFERENCES}

[1] Gonzalez, R.C., Woods, R.E., (2002). Digital Image Processing, second ed. Prentice Hall.

[2] Kim, Y.T., (1997). Contrast enhancement using brightness preserving bi-histogram equalization. IEEE Trans. Consumer Electron. 43 (1), 1-8.

[3] Wan, Y., Chen, Q., Zhang, B.M., (1999). Image enhancement based on equal area dualistic subimage histogram equalization method. IEEE Trans. Consumer Electron. 45 (1), 68-75.

[4] Chen, S.D., Ramli, A.R., (2003a). Contrast enhancement using recursive mean-separate histogram equalization for scalable brightness preservation. IEEE Trans.Consumer Electron. 49 (4), 1301-1309.

[5] Chen, S.D., Ramli, A.R., (2003b). Minimum mean brightness error bi-histogram equalization in contrast enhancement. IEEE Trans. Consumer Electron. 49 (4), 1310-1319.

[6] Sim, K.S., Tso, C.P., Tan, Y.Y., (2007). Recursive sub-image histogram equalization applied to gray scale images. Pattern Recogn. Lett. 28 (10), 1209-1221.

[7] Soong-Der Chen, Abd. Rahman Ramli, (2004). Preserving brightness in histogram equalization based contrast enhancement techniques. Digital Signal Processing 14 (2004) 413-428.

[8] Sayali Nimkar, Sucheta Shrivastava and Sanal Varghese, (2013).CONTRAST Enhancement and brightness preservation using multidecomposition histogram Equalization Signal \& Image Processing : An International Journal (SIPIJ) Vol.4, No.3, June 2013.

[9] Gurvir Sing, Mandeep Singh, (2013). Histogram Eualization Techniques for Image Enhancement using Fuzzy Logic. International Journal of Engineering and Management Research.Volume-3, Issue6, December-2013, ISSN No.: 2250-0758.

[10] Kuldeep Singh, Rajiv Kapoor, (2014) Image enhancement using Exposure based Sub Image Histogram Equalization, Pattern Recognition Letters No. 36, pp10-14.

\section{Authors}

KANIKA KAPOOR

received B.Tech degree in Electronics and Communication, from Maharshi Dayanand University, Haryana in 2013 and M.Tech degree in Electronics and Communication from ITM University, Haryana in 2015. Her research interest includes Image Processing.

\section{SHAVETA ARORA}

received her B.Tech(Hons.) in Instrumentation and Control Engineering from Kurukshetra University, Haryana(India) in 2001and M.Tech from Punjab Technical University in 2007. She is currently pursuing PhD in Fuzzy Image processing. She is working as Assistant Professor (senior scale) in ITM University. Her expertise areas include Digital Signal Processing, Biomedical Engineering, Non-linear Control system, Image Processing, Fuzzy Logic, Digital Control System and Fuzzy Control System. She has presented and published several research papers in the national and international conferences and journals. She is member of IEEE and ISTE.
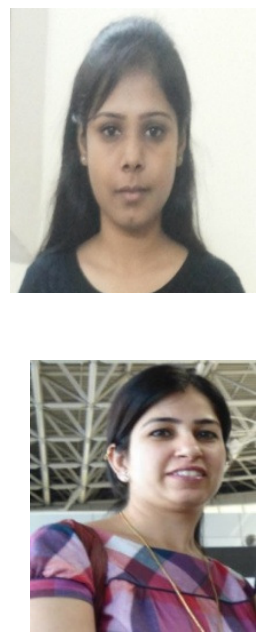